\title{
Ternary functionalised carbon nanofiber/polypyrrole/manganese oxide as high specific energy electrode for supercapacitor
}

\begin{abstract}
A successful fabrication of ternary functionalised carbon nanofibers/polypyrrole/manganese oxide (f-CNFs/PPy/MnO 2 ) composite was reported. The field emission scanning electron microscopy (FESEM) image had revealed a random distribution of granular PPy and spherical nanoparticles of $\mathrm{MnO} 2$ on the surface of f-CNFs. The measured contact angle of f-CNFs indicated superhydrophilic nature which can enhance the surface wettability and ionic diffusion. The ternary $\mathrm{f}-\mathrm{CNF} / \mathrm{PPy} / \mathrm{MnO}_{2}$ composite displayed a remarkable specific capacitance of $409.88 \mathrm{~F} / \mathrm{g}$ compared with f-CNFs/ $\mathrm{MnO}_{2}(322.96 \mathrm{~F} / \mathrm{g})$ and f-CNFs/PPy $(290.83$ $\mathrm{F} / \mathrm{g}$ ). The electrochemical properties of $\mathrm{f}-\mathrm{CNFs} / \mathrm{PPy} / \mathrm{MnO}_{2}$ had contributed to the appreciable specific energy of $42.53 \mathrm{Wh} / \mathrm{kg}$ at a specific power of $297.32 \mathrm{~W} / \mathrm{kg}$. The assembled f$\mathrm{CNFs} / \mathrm{PPy} / \mathrm{MnO}_{2}$ composite also showed low resistance of charge transfer (Rct) value $(3.40 \Omega$ ) with a better cycle life ( $86.30 \%$ capacitance retention over 3000 cycles). Hence, ternary f$\mathrm{CNFs} / \mathrm{PPy} / \mathrm{MnO}_{2}$ can be suggested as a high-performance electrode for supercapacitor.
\end{abstract}

Keyword: Superhydrophilic; Carbon nanofibers; Polypyrrole; Manganese oxide; Supercapacitors 